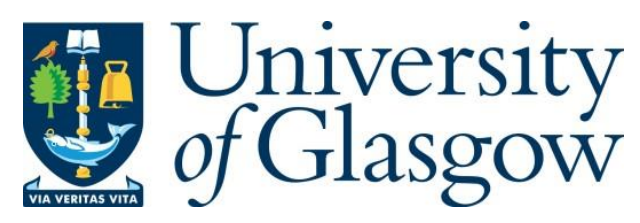

Freeman, E. (2021) Enhancing Ultrasound Haptics with Parametric Audio Effects. In: 23rd ACM International Conference on Multimodal Interaction (ICMI '21), Montréal, QC, Canada, 18-22 Oct 2021, pp. 692-696. ISBN 9781450384810 (doi:10.1145/3462244.3479951).

There may be differences between this version and the published version. You are advised to consult the publisher's version if you wish to cite from it.

(C) The Author 2021. This is the author's version of the work. It is posted here for your personal use. Not for redistribution. The definitive Version of Record was published in Proceedings of the 23rd ACM International Conference on Multimodal Interaction (ICMI '21), Montréal, QC, Canada, 18-22 Oct 2021, pp. 692-696. ISBN 9781450384810 .

http://eprints.gla.ac.uk/249295/

Deposited on: 10 August 2021

Enlighten - Research publications by members of the University of Glasgow http://eprints.gla.ac.uk 


\title{
Enhancing Ultrasound Haptics with Parametric Audio Effects
}

\author{
Euan Freeman \\ University of Glasgow \\ Glasgow, Scotland \\ euan.freeman@glasgow.ac.uk
}

\begin{abstract}
Ultrasound haptic devices can create parametric audio as well as contactless haptic feedback. We investigate if multimodal output from these devices can influence the perception of haptic feedback. We used a magnitude estimation experiment to evaluate perceived roughness of an ultrasound haptic pattern. We found that white noise audio from the haptics device increased perceived roughness and pure tones did not, and that lower rendering frequencies may increase perceived roughness. Our results show that multimodal output has the potential to expand the range of sensations that can be presented by an ultrasound haptic device, paving the way to richer mid-air haptic interfaces.
\end{abstract}

\section{CCS CONCEPTS}

- Human-centered computing $\rightarrow$ Haptic devices; Sound-based input / output; Auditory feedback.

\section{KEYWORDS}

audio-haptic illusion; multisensory perception; ultrasound haptics;

ACM Reference Format:

Euan Freeman. 2021. Enhancing Ultrasound Haptics with Parametric Audio Effects. In Proceedings of the 2021 International Conference on Multimodal Interaction (ICMI '21), October 18-22, 2021, Montréal, QC, Canada. ACM, New York, NY, USA, 5 pages. https://doi.org/10.1145/3462244.3479951

\section{INTRODUCTION}

Ultrasound haptic devices create contactless haptic sensations in mid-air, without the need to wear or touch anything. They focus numerous ultrasound waves to create focal points that are modulated to create a range of sensations. In early work, focal point amplitude was modulated to stimulate rapidly-adapting touch receptors [6, 23]. More recently, focal point position is modulated instead [17, 47-49], creating stronger and more varied tactile sensations.

Ultrasound focal points are the basic unit from which mid-air haptic feedback is created. Numerous rendering techniques have been developed to use focal points for complex haptic feedback designs: e.g., for $2 \mathrm{D}$ and $3 \mathrm{D}$ shapes $[20,28,30]$, movement $[15,50]$, complex surfaces $[4,12]$ and affective experiences $[1,2,36]$. These haptic sensations have been used in a broad range of application areas, as discussed in a recent survey of the technology [42].

Creating convincing fine-grained tactile qualities like roughness is challenging, because the spatial resolution of these devices (focal point size) is determined by output frequency. Most ultrasound

ICMI '21, October 18-22, 2021, Montréal, QC, Canada

(C) 2021 Copyright held by the owner/author(s). Publication rights licensed to ACM

This is the author's version of the work. It is posted here for your personal use. Not for redistribution. The definitive Version of Record was published in Proceedings of the 2021 International Conference on Multimodal Interaction (ICMI '21), October 18-22, 2021, Montréal, QC, Canada, https://doi.org/10.1145/3462244.3479951. haptic devices use $40 \mathrm{kHz}$ sound, where the wavelength is $8.6 \mathrm{~mm}$. This is orders of magnitude higher than the fidelity of tactile perception ( $\mathrm{mm}$ vs $\mu \mathrm{m}[25]$ ), exceeding the threshold for micro-texture elements $(0.2 \mathrm{~mm}$ [27]), making it difficult to create textures by mimicking the fine-grained spatial elements of rough materials.

Tactile perception is known to be affected by cues from other modalities. For example, perception of roughness can be influenced by white noise sound effects during tactile exploration [19, 45, 46]. Ultrasound haptic devices can produce audible output using parametric audio techniques, where an audible signal is modulated onto an ultrasonic carrier wave [5, 40, 52].

We describe an experiment investigating if white noise effects, presented as parametric audio from an ultrasound haptic device, can influence the perceived roughness of a haptic pattern. Our results show that white noise influences perceived roughness, and that patterns rendered at lower frequencies are also reported as feeling rougher. Our findings suggest parametric audio effects, which can be synthesised by an ultrasound haptic device in real time using simple amplitude modulation, can expand the range of tactile sensations from these devices, leading to richer haptic feedback.

\section{BACKGROUND}

\subsection{Ultrasound Haptic Feedback}

Ultrasound haptic devices create tactile feedback without direct contact with the skin, through acoustic radiation pressure. This was first demonstrated by Iwamoto et al. [23], who created a single focal point in mid-air. They modulated its amplitude within peak sensitivity range of the rapidly-adapting mechanoreceptors in the palm (around $250 \mathrm{~Hz}$ ), creating a feeling similar to vibration.

Focal points are building blocks for more complex sensations. Obrist et al. [35] found that points amplitude modulated at $16 \mathrm{~Hz}$ or $250 \mathrm{~Hz}$ could stimulate different touch receptors, leading to varied tactile experiences. Carter et al. [6] developed a method for presenting multiple focal points, creating the sensation of a larger contact area. Wilson et al. [50] used sequential presentation of a single focal point to create the illusion of motion across the skin. Long et al. [30] used focal points to create the outlines of volumetric shapes.

Amplitude modulation helped enable continuous perception of a focal point, because pressure is only perceived at the onset of the signal [23]: so, modulating the amplitude at $250 \mathrm{~Hz}$ means onset is perceived 250 times per second. A recent alternative is spatiotemporal modulation (or lateral modulation), where repeated onset is achieved through continuous focal point movement instead [17, 49]. This enables more complex patterns, using different movement trajectories and speeds to create a range of haptic sensations.

Many haptic rendering methods have been developed to use focal point motion to create expressive mid-air haptic experiences. Freeman et al. [12] rendered geometric features at the intersection 
between the hand and a complex surface as a crude representation of macro-texture elements. Beattie et al. [3,4] showed a more nuanced approach to texture generation, extracting features from images and rendering them dynamically in response to hand movement.

Lines and circles are widely used in mid-air haptic perception studies [14, 15, 17, 18, 22, 48, 49], as rendering them is easy through focal point motion. Ablart et al. [1] studied perceived properties of circles, finding that roughness was affected by rendering frequency. Others have demonstrated "supernatural" effects [32] that exploit the rapid motion and unconstrained trajectories.

Ultrasound haptic feedback is often used to create the feeling of touching mid-air user interface elements [7, 13, 43] and virtual objects [28, 30,31]. Others have created more abstract patterns that evoke a playful or affective response [2, 9, 22, 29, 36]. These designs are achievable with the spatial resolution $(8.6 \mathrm{~mm}$ for $40 \mathrm{kHz}$ ultrasound), because the presence of the haptic pattern is sufficient for the impression of physical contact. However, more sophisticated sensations like texture or material roughness will require a different approach as this spatial resolution is too coarse.

\subsection{Parametric Audio}

Ultrasonic frequencies are inaudible to humans. Ultrasound waves can be manipulated to create audible sound, however. Audible sound waves can be modulated onto an ultrasonic carrier wave using amplitude modulation [52]. As it travels through the air, the carrier wave demodulates into audible sound $[5,40]$. This is called parametric audio and is the principle behind directional speakers like the Audio Spotlight [52]. A result of this phenomena is that sound appears to emanate from a 'virtual' source, rather than the emitter.

When phase is manipulated to focus the sound waves (like for ultrasound haptic focal points), the audible sound can only be heard near the focal point-the virtual source. This was used in Holographic Whisper [37] to create movable audio focal points, targeted near the intended recipient for audio feedback.

Parametric audio can be created using the same hardware as ultrasound haptic feedback. For example, Hirayama et al. [21] used acoustic levitation, parametric audio and ultrasound haptics to create multimodal display content in one two-sided ultrasound device and Martinez-Plasencia et al. [39] developed algorithms to enhance multimodal ultrasonic output from a single device.

There has been little work exploring the perceptual benefits of combining parametric audio and ultrasound haptics. An interesting aspect of this combination is that audio would appear to come from the hand, rather than the ultrasound array. Exploring this is a compelling topic, as it could pave the way to multisensory experiences with richer and more immersive haptics.

\subsection{Roughness}

Roughness is an inherently multisensory sensation: as skin moves across a surface, tactile sensations and movement-produced audio combine to create the notion that the surface has a particular roughness (or smoothness). A key characteristic in surface roughness is the physical size and separation of physical elements: macrotexture and micro-texture elements being greater, and less than, $0.2 \mathrm{~mm}$ respectively [27]. We point readers towards work by Klatzky and Lederman [27], who discuss texture perception in detail, and
Beattie et al. [3], who interpret the literature on roughness with a focus on ultrasound haptic perception. The latter is particularly relevant to this paper, since ultrasound haptic stimuli lack the physical elements that contribute to typical roughness perception.

Tactile roughness can be created with a variety of haptic devices, e.g., vibrotactile actuators $[8,16,44]$, force feedback devices $[33,38]$ and ultrasound haptic devices $[3,4,12]$. These often induce roughness via temporal rather than spatial cues (e.g., since a vibrotactile actuator cannot move or an ultrasound focal point is too large).

\subsection{Audio-Haptic Integration}

Our goal is to combine parametric audio and ultrasound haptic feedback to see if we can expand the range of tactile sensations that can be created by an ultrasound haptics device. Sensory signals from multiple modalities are integrated into a single percept [10]. Information from one modality can influence perception of another when their signals are integrated. Auditory and haptic signals are integrated when exploring a surface or material, partly due to sounds created from mechanical interactions between the surface and the point of contact [27]. Even in the absence of sound, regions of the brain associated with auditory processing are activated when exploring a tactile surface [46].

Several studies have shown that sound can change the perceived tactile roughness of a surface. Guest et al. [19] manipulated the sounds produced by participants' hands moving across abrasive materials; they found that amplifying high frequencies increased perceived roughness and reducing high frequencies decreased perceived roughness. Suzuki et al. [46] found that white noise increased perceived roughness of abrasive materials, but pure tones did not (and similar findings were reported in other studies [26, 45]). In their experiments, participants actively explored that material by moving their fingers back and forth across its surface, in time with an auditory stimulus. The timing stimulus was either a sequence of 1000 beeps, or changes in amplitude of continuous white noise, once per second. We replicate their method, except participants move their hand across an ultrasonic 'surface', not a physical one.

Whilst the cause is unclear, Suzuki et al. [46] hypothesised that white noise has this effect because its broad spectrum contains frequencies associated with touch-produced sounds and amplifying these frequencies leads to a rougher perception [19].

In this paper, we investigate if audio cues can increase perceived roughness of ultrasound haptic output. Texture perception relies on fine-grained spatial cues that are challenging to render using an ultrasound haptic device, as discussed earlier. If auditory effects can influence the perception of ultrasound haptic feedback then this could be an effective means of expanding the range of tactile sensations that can be created with ultrasound haptic devices.

\section{EXPERIMENT}

\subsection{Design and Procedure}

In this experiment we examined if parametric audio effects from an ultrasound haptic device have an effect on perceived roughness of a haptic pattern. We used a within-subjects design with two factors: parametric sound effect (noise, tone, none) and haptic render frequency $(\{50,70,90\} \mathrm{Hz})$. Our baseline condition is no parametric audio effect (i.e., only haptic feedback will be presented). We 


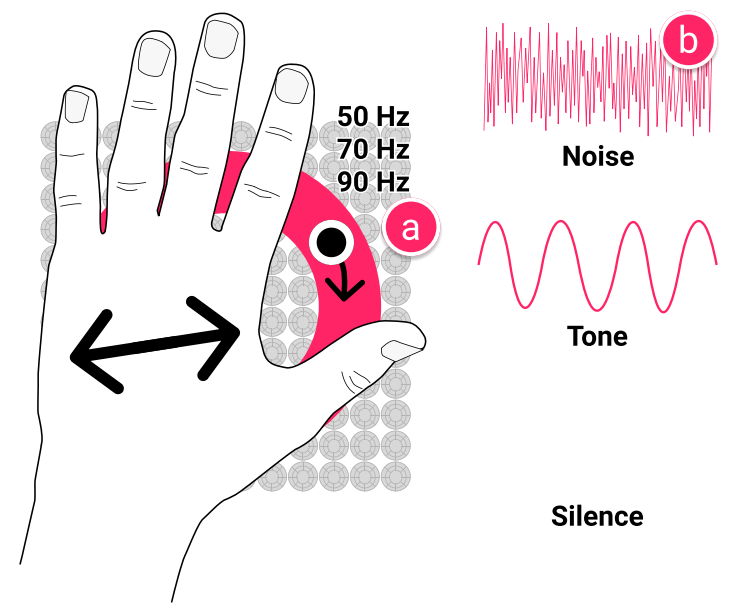

Figure 1: We rendered a circular haptic pattern using a focal point moving at one of three frequencies (a): 50, 70, $90 \mathrm{~Hz}$. This was accompanied by a parametric audio effect (b): noise, tone, or none. Participants moved their hand back and forth across the haptic pattern then rated how rough it felt.

compare this with white noise and a pure sine tone, each presented directly from the ultrasound haptic device. Haptic render frequency determines how many haptic patterns are rendered per second. We varied render frequency because we needed to deceive participants into thinking the haptic patterns did, indeed, vary in roughness. Each of the nine conditions was repeated eight times, for a total of 72 trials. Trials were presented in a random order.

For each trial, we presented one stimuli and asked participants to move their hand back and forth across it (like in [46]). Each stimuli was presented for 10 seconds. We used magnitude estimation to collect judgements of perceived roughness. Magnitude estimation is commonly used to estimate roughness [27], and has also been used to estimate ultrasound haptic roughness [3] and intensity [17, 18]

After each trial, we asked participants to report its roughness using a numerical scale of their own choosing, as recommended by Jones and Tan [24]. Roughness estimates were normalised, since participants used their own rating scales. We divided the estimates from each person by the geometric mean of their own responses [24], then normalised to a range from $0-1$, where 1 was the highest roughness estimate by that person. We asked participants to report haptic and audio roughness separately, to encourage them to focus on the haptics (rather than potential cross-talk or auditory dominance). If they did not feel anything, a zero rating was recorded. The next trial started when the participant indicated they were ready (but no sooner than 10 seconds after the previous trial).

We recruited 13 participants who had never used an ultrasound haptic device before. Six were female, seven were male, all were right handed, mean age 27.5 years (SD 7.1 years).

\subsection{Implementation}

We used an Ultraleap UHEV1 device, which has $25640 \mathrm{kHz}$ emitters. We created a $4 \mathrm{~cm}$ diameter circle pattern, using a rapidly moving focal point (using spatiotemporal modulation [17, 49]). We chose

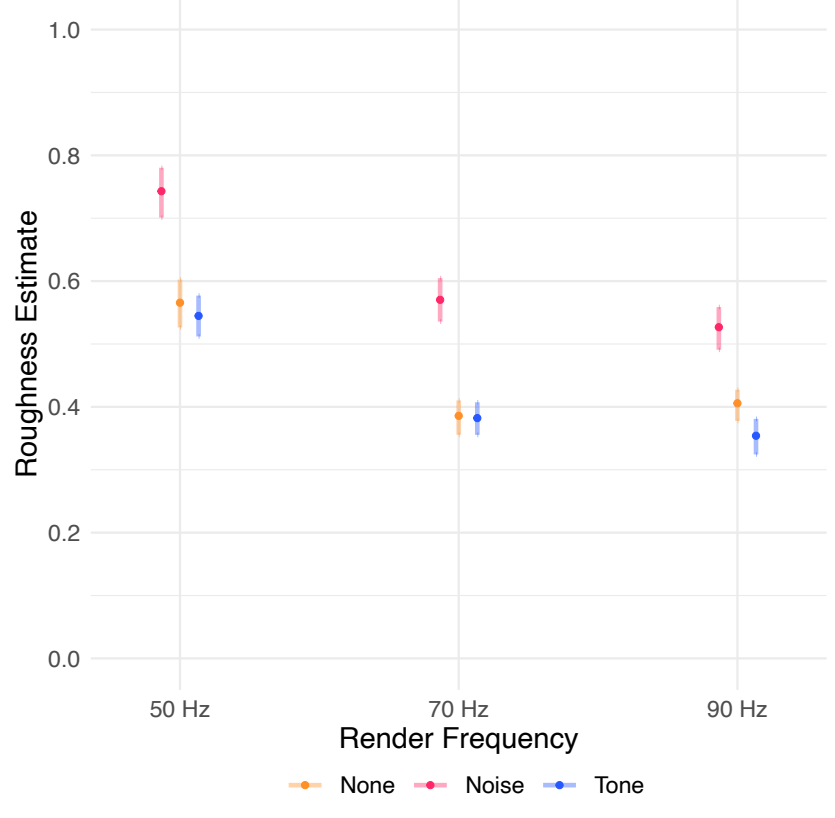

Figure 2: Mean roughness estimates with 95\% CIs.

a circular trajectory because it allows the focal point to move at a uniform speed without any change in direction (similar to other perception experiments, e.g., $[17,18])$. This feels like a surface when the palm moves across it. The circle was in a fixed position $12 \mathrm{~cm}$ above the centre of the device, where feedback is strong [14, 34].

We created audible sound effects from the ultrasound device by creating a separate, fixed-position focal point away from the circle pattern, whose amplitude was modulated at audible frequencies. We synthesised white noise using a random number generator to assign amplitude from $0.0-1.0$, updating at the maximum sample rate of $8 \mathrm{kHz}$ for two points. This produced white noise with a central frequency approx $4 \mathrm{kHz}$. For comparison, we also included a pure tone condition, by synthesising a $1000 \mathrm{~Hz}$ sine wave. We used an experimental version of the UHEV1 firmware that improves parametric audio output, so that audio was less constrained.

We note that creating a secondary focal point for audio may affect the haptic output strength, since sound pressure will be distributed amongst both haptic and auditory points. Since the time-averaged sound pressure is limited for safety, peak output pressure may be below the maximum output pressure (approx $3000 \mathrm{~Pa}$ [34]), so the difference in haptic intensity is less than would be expected.

\subsection{Results}

All data is available online [11]. Figure 2 shows mean haptic roughness estimates for each condition, with $95 \%$ CIs. We do not analyse or report the auditory judgements, as they predictably reflect the difference in audio conditions. A repeated-measures ANOVA found:

- a significant main effect of parametric audio on perceived roughness: $\mathrm{F}(2,96)=121.4, \mathrm{p}<.001, \eta_{p}{ }^{2}=.72$;

- a significant main effect of rendering frequency on perceived roughness: $\mathrm{F}(2,96)=129.9, \mathrm{p}<.001, \eta_{p}^{2}=.73$; 
- no significant interaction effect: $\mathrm{F}(4,96)=1.14, \mathrm{p}=.34$;

Post hoc t-test comparisons for audio found higher roughness estimates for noise vs no audio $(\mathrm{t}=12.5, \mathrm{p}<.001)$ and for noise vs tones $(\mathrm{t}=14.3, \mathrm{p}<.001)$. There was no significant difference for tones vs no audio $(\mathrm{t}=.024, \mathrm{p}=.15)$.

Post hoc $t$-test comparisons for pattern frequency found higher roughness estimates for $50 \mathrm{~Hz}$ vs $70 \mathrm{~Hz}(\mathrm{t}=13.2$, $\mathrm{p}<.001)$ and for $50 \mathrm{~Hz}$ vs $90 \mathrm{~Hz}(\mathrm{t}=14.6, \mathrm{p}<.001)$. There was no difference for $70 \mathrm{~Hz}$ vs $90 \mathrm{~Hz}(\mathrm{t}=1.36, \mathrm{p}=.37)$.

\subsection{Discussion}

Our results suggest white noise from an ultrasound haptic device can make its haptic output feel more rough. Similar findings were observed in other tactile roughness studies [19, 45, 46], although ours is unique because our stimuli were modulated ultrasound rather than abrasive materials. In those studies, noise enhanced the high-frequency sounds caused by the skin moving over the rough surface, leading to the multisensory illusion that the materials were rougher than reality. However, there is no movement-produced sound with an ultrasound haptics device, so our parametric audio effects are the only rough sounds our participants heard (other than audible side effects from the haptic focal point motion).

Despite this, we found a strong effect of white noise on perceived roughness $\left(\eta_{p}^{2}=.72\right)$. We asked participants to rate the roughness of the auditory and haptic stimuli separately, so we are satisfied that they did not confuse the two modalities. However, there may still be an auditory masking or cross-talk effect of the haptic pattern feeling rough because it sounds rough [51]. The cause is likely a combination of these factors: interference across modalities and the stimuli sounding rough. The most important point is that this effect was successful, regardless of whether auditory masking was present, and this was consistent across a range of rendering frequencies.

We also saw a strong effect of rendering frequency on perceived roughness $\left(\eta_{p}^{2}=.73\right)$, with the $50 \mathrm{~Hz}$ pattern considered more rough than the others. This is consistent with findings by Ablart et al. [1], who found that perceived roughness of circles peaks around $25 \mathrm{~Hz}$ and decreases from that point. One explanation for this could be overlap in touch receptors being stimulated by the $50 \mathrm{~Hz}$ pattern. High frequency vibrations $(30-500 \mathrm{~Hz})$ primarily stimulate Pacinian Corpuscles, whereas lower frequencies $(1-50 \mathrm{~Hz})$ primarily stimulate Meissner Corpuscles [25, 41]. Rendering frequency (which identifies focal point movement speed) does not necessarily correspond with the stimulation frequency of a touch receptor. However, in the $50 \mathrm{~Hz}$ conditions, both receptors are likely to be stimulated due to the rate at which focal points pass over receptors. Meissner Corpuscles create a 'fluttering' sensation that may feel rougher than the continuous vibrations felt at higher frequencies $[1,15]$.

Independently, rendering frequency and parametric audio effects can be an effective way of altering the perceived roughness of an ultrasound haptic pattern. When combined, the effect is even more convincing. These are feasible ways of expanding the range of sensations created by an ultrasound haptic device, without modifying focal point trajectory in a spatiotemporal modulated pattern.

Our parametric audio effects were presented continuously from the ultrasound device, which might be undesirable in some contexts. When our hands explore rough materials, roughness is felt during motion, a result of mechanical interference between a rough surface and skin movement [27]. Therefore a compelling area for future work is to investigate if similar effects exist when parametric audio is synthesised dynamically in response to hand motion. This way, the white noise is only audible when the hand is actively exploring a mid-air haptic object, which may be more immersive and acceptable than continuous noise and could enhance the roughness effect.

\section{CONCLUSION}

In this paper, we investigated if parametric audio effects created by an ultrasound haptic device can influence the perception of mid-air haptic feedback. We presented a magnitude estimation experiment investigating the perceived roughness of haptic patterns when accompanied by white noise and pure tone audio effects. This was the first investigation of the perceptual benefits of multimodal output from an ultrasound haptic device.

We found that white noise audio creates the perception of rougher haptic patterns, due to multisensory integration and a potential auditory masking effect: i.e., the idea that haptic patterns feel rough because they sound rough, with sound appearing to be co-located with the hand. Our results also suggest that lower frequencies can lead to perception of increased roughness. Together, these strategies can be used to vary the perceived roughness of a haptic pattern for more expressive output from an ultrasound haptic device. Haptic designers could use this approach to, for example, create user interface elements $[7,13,29,43]$ and virtual objects $[9,31]$ with varying surface textures, for an immersive multimodal experience.

\section{ACKNOWLEDGMENTS}

This research was supported by the European Union's Horizon 2020 research and innovative programme (\#737087: Levitate).

\section{REFERENCES}

[1] Damien Ablart, William Frier, Hannah Limerick, Orestis Georgiou, and Marianna Obrist. 2019. Using Ultrasonic Mid-air Haptic Patterns in Multi-Modal User Experiences. In 2019 IEEE International Symposium on Haptic, Audio and Visual Environments and Games (HAVE). 1-6. https://doi.org/10.1109/HAVE.2019.8920969

[2] Damien Ablart, Carlos Velasco, and Marianna Obrist. 2017. Integrating Mid-Air Haptics into Movie Experiences. In Proceedings of the 2017 ACM International Conference on Interactive Experiences for TV and Online Video - TVX '17. ACM, 77-84. https://doi.org/10.1145/3077548.3077551

[3] David Beattie, William Frier, Orestis Georgiou, Benjamin Long, and Damien Ablart. 2020. Incorporating the Perception of Visual Roughness into the Design of Mid-Air Haptic Textures. In ACM Symposium on Applied Perception 2020 (SAP '20). ACM, Article 4, 10 pages. https://doi.org/10.1145/3385955.3407927

[4] David Beattie, Orestis Georgiou, Adam Harwood, Rory Clark, Benjamin Long, and Tom Carter. 2019. Mid-Air Haptic Textures from Graphics. In Proceedings of the IEEE World Haptics Conference 2019 - Demos.

[5] H. O. Berktay. 1965. Parametric amplification by the use of acoustic non-linearities and some possible applications. Fournal of Sound and Vibration 2, 4 (1965), 462470. https://doi.org/10.1016/0022-460X(65)90123-9

[6] Thomas Carter, Sue Ann Seah, Benjamin Long, Bruce Drinkwater, and Sriram Subramanian. 2013. UltraHaptics: Multi-Point Mid-Air Haptic Feedback for Touch Surfaces. In Proceedings of the 26th Symposium on User Interface Software and Technology - UIST '13. ACM, 505-514. https://doi.org/10.1145/2501988.2502018

[7] Patricia I. Cornelio-Martinez, Silvana De Pirro, Chi Thanh Vi, and Sriram Subramanian. 2017. Agency in Mid-air Interfaces. In Proceedings of the 2017 CHI Conference on Human Factors in Computing Systems - CHI '17. ACM, 2426-2439. https://doi.org/10.1145/3025453.3025457

[8] Heather Culbertson and Katherine J Kuchenbecker. 2017. Ungrounded haptic augmented reality system for displaying roughness and friction. IEEE/ASME Transactions on Mechatronics 22, 4 (2017), 1839-1849.

[9] Brygida Dzidek, William Frier, Adam Harwood, and Richard Hayden. 2018. Design and Evaluation of Mid-Air Haptic Interactions in an Augmented Reality 
Environment. In Proceedings of EuroHaptics 2018 in LNCS 10894 - EuroHaptics '18. Springer, 489-499. https://doi.org/10.1007/978-3-319-93399-3_42

[10] Marc O. Ernst and Martin S. Banks. 2002. Humans integrate visual and haptic information in a statistically optimal fashion. Nature 415, 6870 (2002), 429-433. https://doi.org/10.1038/415429a

[11] Euan Freeman. 2021. User Study Data for "Enhancing Ultrasound Haptics with Parametric Audio Effects". https://doi.org/10.5281/zenodo.5144878

[12] Euan Freeman, Ross Anderson, Julie Williamson, Graham Wilson, and Stephen Brewster. 2017. Textured Surfaces for Ultrasound Haptic Displays. In Proceedings of the 19th ACM International Conference on Multimodal Interaction - ICMI '17 Demos. ACM. https://doi.org/10.1145/3136755.3143020

[13] Euan Freeman, Stephen Brewster, and Vuokko Lantz. 2014. Tactile Feedback for Above-Device Gesture Interfaces: Adding Touch to Touchless Interactions. In Proceedings of the 16th International Conference on Multimodal Interaction - ICMI '14. ACM, 419-426. https://doi.org/10.1145/2663204.2663280

[14] Euan Freeman, Dong-Bach Vo, and Stephen Brewster. 2019. HaptiGlow: Helping Users Position their Hands for Better Mid-Air Gestures and Ultrasound Haptic Feedback. In Proceedings of the IEEE World Haptics Conference 2019. IEEE, 289-294. https://doi.org/10.1109/WHC.2019.8816092

[15] Euan Freeman and Graham Wilson. 2021. Perception of Ultrasound Haptic Focal Point Motion. In Proceedings of the 23rd ACM International Conference on Multimodal Interaction - ICMI '21. ACM. https://doi.org/10.1145/3462244.3479950

[16] Euan Freeman, Graham Wilson, Dong-Bach Vo, Alexander Ng, Ioannis Politis, and Stephen Brewster. 2017. Multimodal Feedback in HCI: Haptics, Non-Speech Audio, and Their Applications. In The Handbook of Multimodal-Multisensor Interfaces: Foundations, User Modeling and Common Modality Combinations.

[17] William Frier, Damien Ablart, Jamie Chilles, Benjamin Long, Marcello Gior dano, Marianna Obrist, and Sriram Subramanian. 2018. Using Spatiotemporal Modulation to Draw Tactile Patterns in Mid-air. In Proc. of EuroHaptics 2018.

[18] William Frier, Dario Pittera, Damien Ablart, Marianna Obrist, and Sriram Subramanian. 2019. Sampling Strategy for Ultrasonic Mid-Air Haptics. In Proceedings of the 2019 CHI Conference on Human Factors in Computing Systems Proceedings CHI '19. ACM, Paper 121. https://doi.org/10.1145/3290605.3300351

[19] Steve Guest, Caroline Catmur, Donna Lloyd, and Charles Spence. 2002. Audiotactile interactions in roughness perception. Experimental Brain Research 146, 2 (2002), 161-171. https://doi.org/10.1007/s00221-002-1164-z

[20] Daniel Hajas, Dario Pittera, Antony Nasce, Orestis Georgiou, and Marianna Obrist. 2020. Mid-Air Haptic Rendering of 2D Geometric Shapes with a Dynamic Tactile Pointer. IEEE Transactions on Haptics 13, 1 (2020), 1-12. https://doi.org/ 10.1109/TOH.2020.2966445

[21] Ryuji Hirayama, Diego Martinez Plasencia, Nobuyuki Masuda, and Sriram Subramanian. 2019. A volumetric display for visual, tactile and audio presentation using acoustic trapping. Nature 575 (2019). https://doi.org/10.1038/s41586-019-1739-5

[22] Thomas Howard, Gerard Gallagher, Anatole Lécuyer, Claudio Pacchierotti, and Maud Marchal. 2019. Investigating the Recognition of Local Shapes Using Mid-air Ultrasound Haptics. Proceedings of the 2019 IEEE World Haptics Conference - WHC '19 (2019), 503-508. https://doi.org/10.1109/WHC.2019.8816127

[23] Takayuki Iwamoto, Mari Tatezono, and Hiroyuki Shinoda. 2008. Non-contact method for producing tactile sensation using airborne ultrasound. In Proceedings of EuroHaptics 2008. Springer. https://doi.org/10.1007/978-3-540-69057-3_64

[24] Lynette A. Jones and Hong Z. Tan. 2013. Application of Psychophysical Techniques to Haptic Research. IEEE Transactions on Haptics 6, 3 (2013), 268-284. https://doi.org/10.1109/TOH.2012.74

[25] Eric R. Kandel, James H. Schwartz, Thomas M. Jessell, Steven A. Siegelbaum, and A. J. Hudspeth. 2012. Touch. In Principles of Neural Science (5th ed.). McGraw-Hill Publishing, Chapter 23, 498-529.

[26] Seung-Chan Kim, Ki-Uk Kyung, and Dong-Soo Kwon. 2007. The Effect of Sound on Haptic Perception. Second foint EuroHaptics Conference and Symposium on Haptic Interfaces for Virtual Environment and Teleoperator Systems - WHC '07 (2007). https://doi.org/10.1109/WHC.2007.110

[27] Roberta L. Klatzky and Susan J. Lederman. 2010. Multisensory Texture Perception. In Multisensory Object Perception in the Primate Brain. Springer, Chapter 12, 211230. https://doi.org/10.1007/978-1-4419-5615-6_12

[28] Georgios Korres and Mohamad Eid. 2016. Haptogram: Ultrasonic Point-Cloud Tactile Stimulation. IEEE Access 4 (2016), 7758 - 7769. https://doi.org/10.1109/ ACCESS.2016.2608835

[29] Hannah Limerick, Richard Hayden, David Beattie, Orestis Georgiou, and Jörg Müller. 2019. User Engagement for Mid-Air Haptic Interactions with Digital Signage. In Proceedings of the 8th ACM International Symposium on Pervasive Displays. ACM, Article 15. https://doi.org/10.1145/3321335.3324944

[30] Benjamin Long, Sue Ann Seah, Tom Carter, and Sriram Subramanian. 2014. Rendering Volumetric Haptic Shapes in Mid-Air using Ultrasound. ACM Transaction on Graphics 33, 6 (2014), Article 181. https://doi.org/10.1145/2661229.2661257

[31] Yasutoshi Makino, Yoshikazu Furuyama, Seki Inoue, and Hiroyuki Shinoda. 2016. HaptoClone (Haptic-Optical Clone) for Mutual Tele-Environment by Real-time 3D Image Transfer with Midair Force Feedback. In Proceedings of the SIGCHI Conference on Human Factors in Computing Systems - CHI '16. ACM, 1980-1990. https://doi.org/10.1145/2858036.2858481
[32] Jonatan Martinez, Daniel Griffiths, Valerio Biscione, Orestis Georgiou, and Tom Carter. 2018. Touchless Haptic Feedback for Supernatural VR Experiences. Proceedings of the 25th IEEE Conference on Virtual Reality and 3D User Interfaces (2018), 629-630. https://doi.org/10.1109/VR.2018.8446522

[33] Marilyn McGee, Philip Gray, and Stephen Brewster. 2001. Feeling rough: multimodal perception of virtual roughness. In Proceedings of Eurohaptics '01. 29-33.

[34] Rafael Morales González, Asier Marzo, Euan Freeman, William Frier, and Orestis Georgiou. 2021. UltraPower: Powering Tangible \& Wearable Devices with Focused Ultrasound. In Proceedings of the 15th International Conference on Tangible, Embedded, and Embodied Interaction. ACM. https://doi.org/10.1145/3430524.3440620

[35] Marianna Obrist, Sue Ann Seah, and Sriram Subramanian. 2013. Talking about Tactile Experiences. In Proc. of the SIGCHI Conference on Human Factors in Computing Systems. ACM, 1659-1668. https://doi.org/10.1145/2470654.2466220

[36] Marianna Obrist, Sriram Subramanian, Elia Gatti, Benjamin Long, and Thomas Carter. 2015. Emotions Mediated Through Mid-Air Haptics. In Proceedings of the SIGCHI Conference on Human Factors in Computing Systems - CHI '15. ACM, 2053-2062. https://doi.org/10.1145/2702123.2702361

[37] Yoichi Ochiai, Takayuki Hoshi, and Ippei Suzuki. 2017. Holographic Whisper: Rendering Audible Sound Spots in Three-dimensional Space by Focusing Ultrasonic Waves. In Proceedings of the 2017 CHI Conference on Human Factors in Computing Systems - CHI '17. ACM, 4314-4325. https://doi.org/10.1145/3025453.3025989

[38] Dessislava Peeva, Bridget Baird, Ozgur Izmirli, and Donald Blevins. 2004. Haptic and sound correlations: Pitch, loudness and texture. In Proceedings. Eighth International Conference on Information Visualisation, 2004. IV 2004. IEEE, 659-664.

[39] Diego Martinez Plasencia, Ryuji Hirayama, Roberto Montano-Murillo, and Sriram Subramanian. 2020. GS-PAT: High-Speed Multi-Point Sound-Fields for Phased Arrays of Transducers. ACM Transactions on Graphics 39, 4, Article 138 (2020), 12 pages. https://doi.org/10.1145/3386569.3392492

[40] F. Joseph Pompei. 2002. Sound from ultrasound: the parametric array as an audible sound source. $\mathrm{PhD}$ Thesis. Massachusetts Institute of Technology.

[41] D. Purves, G. J. Augustine, D. Fitzpatrick, W. C. Hall, A.-S. LaMantia, J. O. McNamara, and S. M. Williams (Eds.). 2004. Neuroscience (3rd ed.). Sinauer Associates, Inc. https://doi.org/10.1212/01.WNL.0000154473.43364.47

[42] Ismo Rakkolainen, Euan Freeman, Antti Sand, Roope Raisamo, and Stephen Brewster. 2020. A Survey of Mid-Air Ultrasound Haptics and Its Applications. IEEE Transactions on Haptics (2020). https://doi.org/10.1109/TOH.2020.3018754

[43] Sonja Rümelin, Thomas Gabler, and Jesper Bellenbaum. 2017. Clicks are in the Air: How to Support the Interaction with Floating Objects through Ultrasonic Feedback. In Proceedings of AutomotiveUI '17. ACM, 103-108. https://doi.org/10. 1145/3122986.3123010

[44] Paul Strohmeier and Kasper Hornbæk. 2017. Generating haptic textures with a vibrotactile actuator. In Proceedings of the 2017 CHI Conference on Human Factors in Computing Systems. 4994-5005.

[45] Yuika Suzuki and Jiro Gyoba. 2009. Effects of Sounds on Tactile Roughness Depend on the Congruency between Modalities. In Proceedings of the IEEE World Haptics Conference. IEEE, 150-153. https://doi.org/10.1109/WHC.2009.4810857

[46] Yuika Suzuki, Jiro Gyoba, and Shuichi Sakamoto. 2008. Selective effects of auditory stimuli on tactile roughness perception. Brain Research 1242 (2008), 87-94. https://doi.org/10.1016/j.brainres.2008.06.104

[47] Ryoko Takahashi, Keisuke Hasegawa, and Hiroyuki Shinoda. 2018. Lateral Modulation of Midair Ultrasound Focus for Intensified Vibrotactile Stimuli. In Proceedings of EuroHaptics 2018 in LNCS 10894 - EuroHaptics '18. Springer International Publishing, 276-288. https://doi.org/10.1007/978-3-319-93399-3_25

[48] Ryoko Takahashi, Keisuke Hasegawa, and Hiroyuki Shinoda. 2019. Tactile Stimulation by Repetitive Lateral Movement of Midair Ultrasound Focus. IEEE Transactions on Haptics (2019). https://doi.org/10.1109/TOH.2019.2946136

[49] Ryoko Takahashi, Keisuke Hasegawa, and Hiroyuki Shinoda. 2020. Tactile Stimulation by Repetitive Lateral Movement of Midair Ultrasound Focus. IEEE Transactions on Haptics 13, 2 (2020), 334-342. https://doi.org/10.1109/TOH.2019.2946136

[50] Graham Wilson, Tom Carter, Sriram Subramanian, and Stephen Brewster. 2014. Perception of Ultrasonic Haptic Feedback on the Hand: Localisation and Apparent Motion. In Proceedings of the SIGCHI Conference on Human Factors in Computing Systems - CHI '14. ACM, 1133-1142. https://doi.org/10.1145/2556288.2557033

[51] Jeffrey M Yau, Jonathon B Olenczak, John F Dammann, and Sliman J Bensmaia. 2009. Temporal frequency channels are linked across audition and touch. Current biology 19, 7 (2009), 561-566.

[52] Masahide Yoneyama, Jun-ichiroh Fujimoto, Yu Kawamo, and Shoichi Sasabe. 1983. The audio spotlight: An application of nonlinear interaction of sound waves to a new type of loudspeaker design. The Journal of the Acoustical Society of America 73, 5 (1983), 1532-1536. https://doi.org/10.1121/1.389414 